\title{
The Investigation of Channel Selection Effects on Epileptic Analysis of EEG Signals
}

\author{
M. Yıldız, and E. Bergil
}

\begin{abstract}
A great number of methods are used in order to increase the speed of decision units in epileptic analysis of the multi-channel EEG signals. Channel selection is one of the main methods used for the reduction of the processing load. By eliminating the non-distinct channels, the performance of the system can be improved. In this study, the seizure detection performances of EEG signals obtained by 21 different channels were evaluated. This study was carried out patient-specifically for each six patients. The feature set is generated via calculating 26 features from EEG signals. The dimension of feature set for each channel is reduced using Principal Component Analysis. The reduced feature sets were divided as training and testing data using cross-validation method. With Linear Discriminant Analysis, the classification was done for each channel and performances of channel were compared. Depending on the channel selection, almost $9 \%$ differences in the classification accuracies have been observed.
\end{abstract}

Index Terms-Epilepsy, seizure detection, channel selection, classification.

\section{INTRODUCTION}

$\mathrm{E}$ PILEPSY is a disease that is characterized by recurrent ${ }_{\text {seizures. It occurs without impulse and causes disturbance }}$ in the nervous system. Seizures, can be detected with clinical symptoms, are the temporary anomalies of the electrical activities caused by a group cell in brain. EEG signal has been widely used in the study and diagnosis of epilepsy. The EEG signals can be obtained by placing necessary electrode from different centers and direct measures [1,2,3]. Electrode placement, used the most widely for Multi-channel EEG measurement, is the 10-20 system. Electrode placement for extended 10-20 system is shown in the Figure 1.

Getting started to use the high-performance processors has caused to raise the interest to the issues such as the emergence of seizure phase, detection, and prediction [4]. For the purpose of getting the most meaningful performance in EEG analysis, it is necessary to get the desired signal among multi-channel

M. Yıldı, Electrical-Electronics Engineering Department, Faculty of Engineering,Sakarya University, , P.O. Box 54187 , Sakarya, Turkey, (e-mail: myildiz@sakarya.edu.tr).

E. Bergil, The Program ofControl and Automation Technology,Electronics and Automation Department, Technical Sciences Vocational School, Amasya University, P.O. Box 05100, Amasya, Turkey, (e-mail: erhan.bergil@amasya.edu.tr). measurements. In addition, the signals containing unnecessary information and noise have decrease the performance. Moreover, they cause to lower the reaction speed of the system [5].

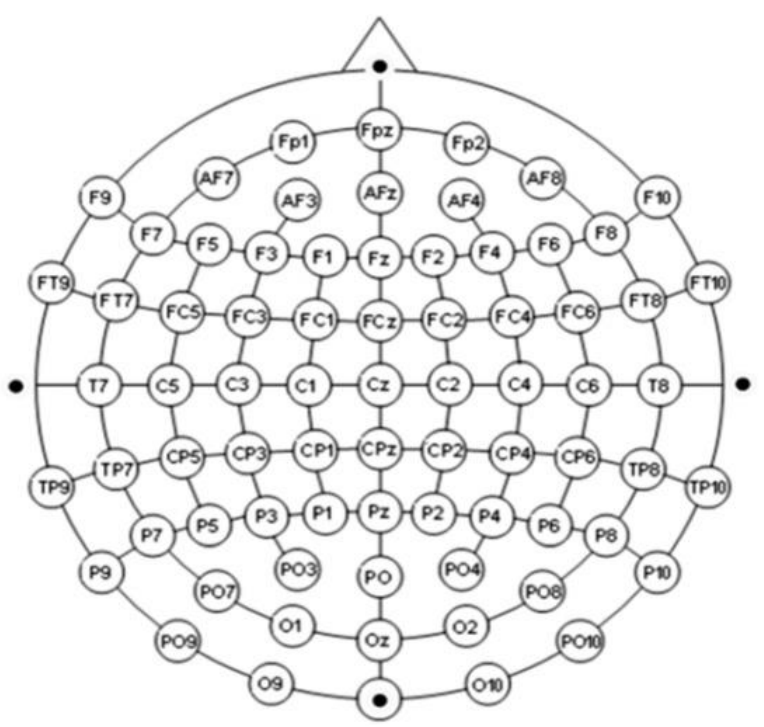

Fig.1. Extended 10/20 system on the cap

Qaraqe et al., have suggested a new architecture to develop the performance of seizure detector. In their studies carried out for patient-specific, they have improved the system sensitivity at $7 \%$ higher than the current ones by utilizing channel selection and feature enhancement. They also have decreased the number of the fault detection appeared per hour [6].

Duun-Henriksen et al., have examined the effects of different channel selection methods on the performance of seizure detection. In their study where they extracted features by using wavelet transform, they used support vector machines as classifier. They reached to the best achievement by the method of maximum variance based channel selection instead of focal channels used extensively. Using only 3 channels, they performed seizure detection without performance reduction [7].

Faul and Marnane have developed dynamic electrode selection method to decrease power consumption in seizure detection. They have provided power saving at $47 \%$ without any decrease in detection performance in the study where they evaluated power consumption and detector performance by using different number of electrodes [8].

Tekgul et al., have performed seizure detection via EEG records taken from newborns with the 10-20 electrode system. 
As a result of the classification performed by using all the electrodes, they have detected the all 31 seizures. They repeated the all processes by using only 9 electrodes. Although they couldn't detect only one patient's seizure for the reduced montage, they reached $100 \%$ specificity and $96,8 \%$ sensitivity respectively comparing with the results taken by using all electrodes [9].

Channel selection process was frequently used to decrease the load of the processor especially in real time applications. With the channel selection process, it was aimed to eliminate the channels not including distinguishing information. By this way, it is possible to design high-speed detectors without losing performance in the process of seizure detection. In this study, the effects of EEG signals taken from different channels on epileptic analysis have been evaluated. The multi-channel EEG signals taken from 6 epilepsy patients have been analyzed. Since the characteristics of epilepsy differs patient specifically, the analysis have been done in accordance to the patient specifically. At the beginning, feature sets from normal and seizure stage EEG signals for each channel have been generated. In the following step, the dimension of these feature sets has been decreased via Principal Component Analysis (PCA). 26 features were reduced to 8 features. The feature set for each channel has been divided into 2 parts as testing and training data by the method of cross-validation. Via Linear Discriminant Analysis (LDA), classification process has been carried out for 21 different channels and their classification accuracies have been compared. In consequences of the analysis, it is aimed to contribute to the design and production studies of portable seizure detection systems by determining the effects of channel on epileptic analysis.

\section{MATERIAL AND METHOD}

In this study, the process consists of pre-processing, feature extraction, dimension reduction, PCA distribution and classification stages. First of all, pre-processing where the EEG signals taken from normal and seizure phases divided into 5 second parts has been performed. In the second stage, feature set has been obtained by calculating 26 features for each EEG period. Feature set has been calculated for different 21 channels. Then, the number of the features has been decreased to 8 by PCA method. With cross-validation method, testing and training data have been obtained and by this way the classification process has been performed. LDA method has been used for classifier. The classification process has been performed by using 1000 different testing and training data. The classification results and data of testing and training have been registered for the highest classification performance. In order to observe the effect of channels on classification performance, PCA projection has been used. The testing data of channels having the highest and lowest accuracy which belongs to the patient 4 has been reflected to the plane consisting of the highest weight first two principal components and the principal component distribution has been obtained. The block schema of this process has been illustrated in the Figure 2.

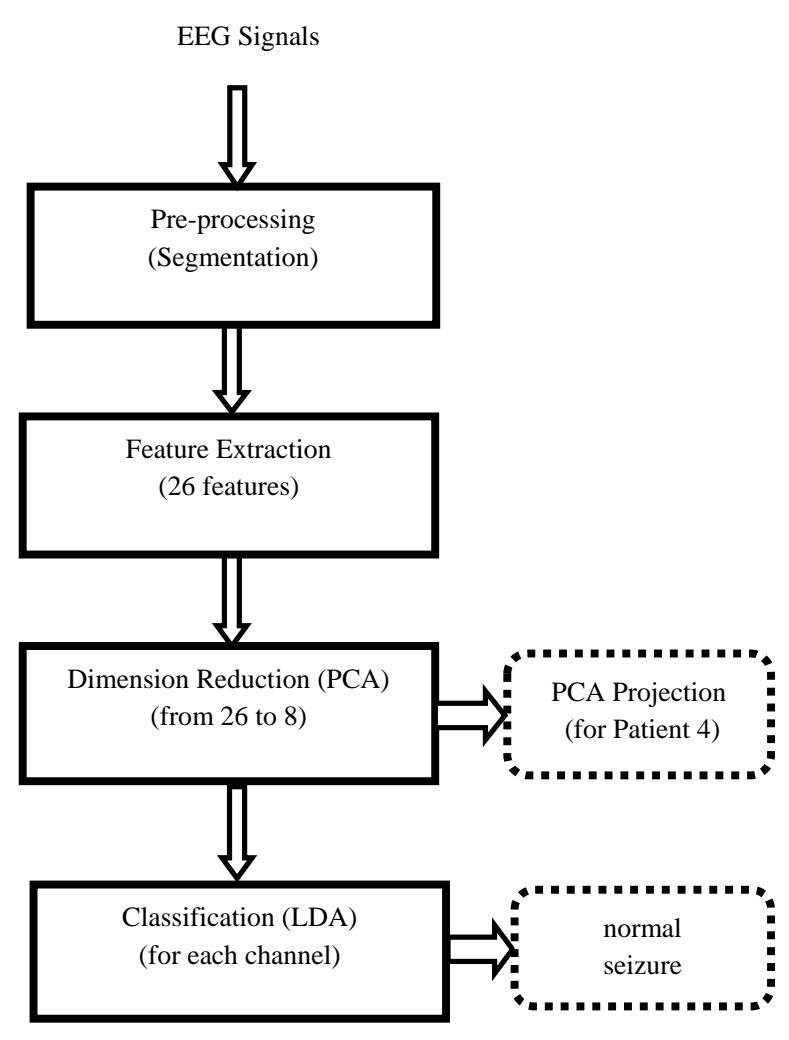

Fig.2. Block diagram of the process

EEG signals were taken from PhysioNet database [10]. The scalp EEG signals were obtained using 10-20 electrode system and sampled in $256 \mathrm{~Hz}$. The starting points and total durations of seizures in EEG records were remarked by the specialists. Data, taken from seizure and seizure-free stages, were divided into 5 second periods. This study was done for 6 epilepsy patients. The demographic information of patients is given in the Table I.

TABLE I

DEMOGRAPHIC INFORMATION

\begin{tabular}{|c|c|c|}
\hline Patient & Sex & Age \\
\hline Patient 1 & Female & 11 \\
\hline Patient 2 & Female & 14 \\
\hline Patient 3 & Female & 15 \\
\hline Patient 4 & Male & 3.5 \\
\hline Patient 5 & Male & 3 \\
\hline Patient 6 & Female & 6 \\
\hline
\end{tabular}

The numbers of sample belong to normal and seizure stage for all patient are given in the Table II. 
TABLE II

THE SAMPLE NUMBERS FOR EACH PATIENT

\begin{tabular}{|c|c|c|}
\hline Patient & Normal Stage & Seizure Stage \\
\hline Patient 1 & 192 & 88 \\
\hline Patient 2 & 192 & 78 \\
\hline Patient 3 & 192 & 108 \\
\hline Patient 4 & 192 & 182 \\
\hline Patient 5 & 192 & 88 \\
\hline Patient 6 & 192 & 84 \\
\hline
\end{tabular}

Feature Extraction: 26 features widely used in epilepsy studies are selected. These features, based on time-domain, frequencydomain and power spectrum, are given in the Table III.

\begin{tabular}{|c|c|c|c|}
\multicolumn{5}{|c|}{ TABLE III } \\
\hline No & Feature & No & Feature \\
\hline 1 & Mean & 11 & Hjorth Activity \\
\hline 2 & Standard Deviation & 12 & Hjhort Mobility \\
\hline 3 & Variance & 13 & Hjhort Complexity \\
\hline 4 & Total Power & 14 & Renyi Entropy \\
\hline 5 & $\begin{array}{c}\text { Power of Delta Band } \\
(0.5-2 \text { Hz) }\end{array}$ & 15 & Entropy \\
\hline 6 & $\begin{array}{c}\text { Power of Delta Band } \\
(2-4 \text { Hz) }\end{array}$ & 16 & Maximum \\
\hline 7 & Power of Theta Band & 17 & Minimum \\
\hline 8 & Power of Alpha Band & 18 & Zero Cross Rate \\
\hline 9 & Power of Beta Band & $19-26$ & $\begin{array}{c}\text { Auto-Regressive } \\
\text { Coefficients (Burg Method) }\end{array}$ \\
\hline 10 & Mod & & \\
\hline
\end{tabular}

Dimension Reduction:26 features generated from EEG signals have been decreased to 8 with the method of Principal Component Analysis. The studies of principal component analysis started with Karl Pearson in 1901 were developed by Hotelling in 1933. It is a transform technique which uses to convert a data set of correlated variables into a set of linearly uncorrelated variables. This method can be used for dimension reduction. The variables after the transformation are called as principal components of first variables. The first principal component has the highest variance value and the other principal components are in the decreasing variance values order. Low sensitivity towards noise, the decrease of the memory and capacity needs can be mentioned among its main advantages [11,12].

Classification: In classification stage of this study, Linear Discrimination Analysis has been performed. The feature set has been divided into testing and training data by crossvalidation method for each patient. At the end of this classification process, it was aimed to detect the normal and seizure stage of the testing data.

Linear Discriminant Analysis is one of the classification methods that is commonly used in the fields of machine learning and statistics. LDA is a method of which features can be used as linear classifiers to separate the samples of two or more classes and which tries to find the linear combination. In other words, LDA is a method, tries in order to get vectors belong to the space, which are able to separate each classes [1317].

This approach generates a new variable which is the combination of the available data. This variable closers to data points in the same class each other, and pushes apart the data points belonging to different classes [14,16,17].

\section{RESULTS OBTAINED AND DISCUSSION}

The all feature sets have been divided into training and testing data with the cross-validation method. Afterwards, classification has been performed by using LDA. This process was repeated 1000 times for 21 channels, the training and testing data that have the highest accuracy classification performance was registered. As a result of this processes, the best classification performances belonging to 6 patients for 21 different channels have been shown in the tables IV,V,VI,VII,VIII and IX. In the tables, the channels showing the worst classification performances have been highlighted.

TABLE IV

THE CLASSIFICATION RESULTS FOR PATIENT 1

\begin{tabular}{|c|c|c|c|c|c|}
\hline \multirow{2}{*}{ Channel } & \multicolumn{2}{|c|}{ normal stage } & \multicolumn{2}{c|}{ seizure stage } & \multirow{2}{*}{ Accuracy } \\
\cline { 2 - 5 } & normal & seizure & normal & seizure & \\
\hline Fp1-F7 & 64 & 0 & 0 & 29 & 100 \\
\hline F7-T7 & 64 & 0 & 0 & 29 & 100 \\
\hline T7-P7 & 64 & 0 & 0 & 30 & 100 \\
\hline P7-O1 & 64 & 0 & 0 & 29 & 100 \\
\hline Fp1-F3 & 64 & 0 & 0 & 29 & 100 \\
\hline F3-C3 & 64 & 0 & 0 & 30 & 100 \\
\hline C3-P3 & 64 & 0 & 0 & 29 & 100 \\
\hline P3-O1 & 64 & 0 & 0 & 29 & 100 \\
\hline Fp2-F4 & 64 & 0 & 0 & 29 & 100 \\
\hline F4-C4 & 64 & 0 & 0 & 29 & 100 \\
\hline C4-P4 & 64 & 0 & 0 & 29 & 100 \\
\hline P4-O2 & 64 & 0 & 0 & 30 & 100 \\
\hline Fp2-F8 & 64 & 0 & 0 & 29 & 100 \\
\hline F8-T8 & 64 & 0 & 0 & 30 & 100 \\
\hline T8-P8 & 64 & 0 & 0 & 29 & 100 \\
\hline P8-O2 & $\mathbf{6 4}$ & $\mathbf{0}$ & $\mathbf{1}$ & $\mathbf{2 8}$ & $\mathbf{9 8 , 9 2}$ \\
\hline Fz-Cz & 64 & 0 & 0 & 30 & 100 \\
\hline Cz-Pz & 64 & 0 & 0 & 29 & 100 \\
\hline T7-Ft9 & 64 & 0 & 0 & 30 & 100 \\
\hline Ft9-Ft10 & 64 & 0 & 0 & 29 & 100 \\
\hline Ft10-T8 & $\mathbf{6 3}$ & $\mathbf{1}$ & $\mathbf{0}$ & $\mathbf{2 9}$ & $\mathbf{9 8 , 9 2}$ \\
\hline & & & & & \\
\hline & & 0 & 0 & 29 \\
\hline
\end{tabular}


TABLE V

THE CLASSIFICATION RESULTS FOR PATIENT 2

\begin{tabular}{|c|c|c|c|c|c|}
\hline \multirow{2}{*}{ Channel } & \multicolumn{2}{|c|}{ normal stage } & \multicolumn{2}{|c|}{ seizure stage } & \multirow{2}{*}{ Accuracy } \\
\cline { 2 - 5 } & normal & seizure & normal & seizure & \\
\hline Fp1-F7 & 64 & 0 & 0 & 26 & 100 \\
\hline F7-T7 & 64 & 0 & 0 & 26 & 100 \\
\hline T7-P7 & 64 & 0 & 0 & 26 & 100 \\
\hline P7-O1 & 64 & 0 & 0 & 26 & 100 \\
\hline Fp1-F3 & 64 & 0 & 0 & 26 & 100 \\
\hline F3-C3 & 64 & 0 & 0 & 26 & 100 \\
\hline C3-P3 & 64 & 0 & 0 & 26 & 100 \\
\hline P3-O1 & 64 & 0 & 0 & 26 & 100 \\
\hline Fp2-F4 & 64 & 0 & 0 & 26 & 100 \\
\hline F4-C4 & 64 & 0 & 0 & 26 & 100 \\
\hline C4-P4 & 64 & 0 & 0 & 26 & 100 \\
\hline P4-O2 & 64 & 0 & 0 & 26 & 100 \\
\hline Fp2-F8 & 64 & 0 & 0 & 26 & 100 \\
\hline F8-T8 & $\mathbf{6 3}$ & $\mathbf{1}$ & $\mathbf{0}$ & $\mathbf{2 6}$ & $\mathbf{9 8 , 8 9}$ \\
\hline T8-P8 & 64 & 0 & 0 & 26 & 100 \\
\hline P8-O2 & 64 & 0 & 0 & 26 & 100 \\
\hline Fz-Cz & 64 & 0 & 0 & 26 & 100 \\
\hline Cz-Pz & 64 & 0 & 0 & 26 & 100 \\
\hline T7-Ft9 & 64 & 0 & 0 & 26 & 100 \\
\hline Ft9-Ft10 & 64 & 0 & 0 & 26 & 100 \\
\hline Ft10-T8 & 64 & 0 & 0 & 26 & 100 \\
\hline
\end{tabular}

TABLE VI

THE CLASSIFICATION RESULTS FOR PATIENT 3

\begin{tabular}{|c|c|c|c|c|c|}
\hline \multirow{2}{*}{ Channel } & \multicolumn{2}{|c|}{ normal stage } & \multicolumn{2}{|c|}{ seizure stage } & \multirow{2}{*}{ Accuracy } \\
\cline { 2 - 5 } & normal & seizure & normal & seizure & \\
\hline Fp1-F7 & 62 & 2 & 0 & 36 & 98 \\
\hline F7-T7 & 63 & 1 & 2 & 34 & 97 \\
\hline T7-P7 & $\mathbf{6 3}$ & $\mathbf{1}$ & $\mathbf{3}$ & $\mathbf{3 3}$ & $\mathbf{9 6}$ \\
\hline P7-O1 & 64 & 0 & 2 & 34 & 98 \\
\hline Fp1-F3 & 63 & 1 & 2 & 34 & 97 \\
\hline F3-C3 & 64 & 0 & 0 & 36 & 100 \\
\hline C3-P3 & 64 & 0 & 0 & 36 & 100 \\
\hline P3-O1 & 64 & 0 & 0 & 36 & 100 \\
\hline Fp2-F4 & 63 & 1 & 2 & 34 & 97 \\
\hline F4-C4 & 64 & 0 & 1 & 35 & 99 \\
\hline C4-P4 & 64 & 0 & 0 & 36 & 100 \\
\hline P4-O2 & 64 & 0 & 0 & 36 & 100 \\
\hline Fp2-F8 & 64 & 0 & 0 & 36 & 100 \\
\hline F8-T8 & 64 & 0 & 3 & 33 & 97 \\
\hline T8-P8 & 61 & 3 & 0 & 36 & 97 \\
\hline P8-O2 & 63 & 1 & 0 & 36 & 99 \\
\hline Fz-Cz & 64 & 0 & 0 & 36 & 100 \\
\hline Cz-Pz & 64 & 0 & 0 & 36 & 100 \\
\hline T7-Ft9 & 64 & 0 & 2 & 34 & 98 \\
\hline Ft9-Ft10 & 64 & 0 & 0 & 36 & 100 \\
\hline Ft10-T8 & 64 & 0 & 2 & 34 & 98 \\
\hline
\end{tabular}

TABLE VII

THE CLASSIFICATION RESULTS FOR PATIENT 4

\begin{tabular}{|c|c|c|c|c|c|}
\hline \multirow{2}{*}{ Channel } & \multicolumn{2}{|c|}{ normal stage } & \multicolumn{2}{|c|}{ seizure stage } & \multirow{2}{*}{ Accuracy } \\
\cline { 2 - 5 } & normal & seizure & normal & seizure & \\
\hline Fp1-F7 & 61 & 3 & 3 & 58 & 95,2 \\
\hline F7-T7 & 61 & 3 & 0 & 61 & 97,6 \\
\hline T7-P7 & 62 & 2 & 1 & 60 & 97,6 \\
\hline P7-O1 & 64 & 0 & 1 & 60 & 99,2 \\
\hline Fp1-F3 & $\mathbf{6 0}$ & $\mathbf{4}$ & $\mathbf{7}$ & $\mathbf{5 4}$ & $\mathbf{9 1 , 2}$ \\
\hline F3-C3 & 61 & 3 & 7 & 53 & 91,94 \\
\hline C3-P3 & 63 & 1 & 6 & 55 & 94,4 \\
\hline P3-O1 & 61 & 3 & 0 & 61 & 97,6 \\
\hline Fp2-F4 & 63 & 1 & 4 & 57 & 96 \\
\hline F4-C4 & 63 & 1 & 0 & 61 & 99,2 \\
\hline C4-P4 & 61 & 3 & 0 & 61 & 97,6 \\
\hline P4-O2 & 64 & 0 & 0 & 61 & 100 \\
\hline Fp2-F8 & 60 & 4 & 0 & 61 & 96,8 \\
\hline F8-T8 & 64 & 0 & 0 & 60 & 100 \\
\hline T8-P8 & 64 & 0 & 0 & 60 & 100 \\
\hline P8-O2 & 63 & 1 & 0 & 60 & 99,19 \\
\hline Fz-Cz & 63 & 1 & 1 & 59 & 98,39 \\
\hline Cz-Pz & 64 & 0 & 2 & 59 & 98,4 \\
\hline T7-Ft9 & 64 & 0 & 3 & 58 & 97,6 \\
\hline Ft9-Ft10 & 61 & 3 & 2 & 59 & 96 \\
\hline Ft10-T8 & 64 & 0 & 2 & 59 & 98,4 \\
\hline & & & & & \\
\hline
\end{tabular}

TABLE VIII

THE CLASSIFICATION RESULTS FOR PATIENT 5

\begin{tabular}{|c|c|c|c|c|c|}
\hline \multirow{2}{*}{ Channel } & \multicolumn{2}{|c|}{ normal stage } & \multicolumn{2}{c|}{ seizure stage } & \multirow{2}{*}{ Accuracy } \\
\cline { 2 - 5 } & normal & seizure & normal & seizure & \\
\hline Fp1-F7 & 64 & 0 & 2 & 27 & 97,85 \\
\hline F7-T7 & 64 & 0 & 1 & 29 & 98,94 \\
\hline T7-P7 & 64 & 0 & 0 & 29 & 100 \\
\hline P7-O1 & 64 & 0 & 0 & 29 & 100 \\
\hline Fp1-F3 & 63 & 1 & 1 & 28 & 97,85 \\
\hline F3-C3 & 64 & 0 & 3 & 27 & 96,81 \\
\hline C3-P3 & 64 & 0 & 1 & 29 & 98,94 \\
\hline P3-O1 & 64 & 0 & 0 & 30 & 100 \\
\hline Fp2-F4 & 64 & 0 & 3 & 26 & 96,77 \\
\hline F4-C4 & 64 & 0 & 1 & 28 & 98,92 \\
\hline C4-P4 & 64 & 0 & 0 & 29 & 100 \\
\hline P4-O2 & 64 & 0 & 0 & 30 & 100 \\
\hline Fp2-F8 & 63 & 1 & 3 & 27 & 95,74 \\
\hline F8-T8 & 64 & 0 & 0 & 29 & 100 \\
\hline T8-P8 & 64 & 0 & 2 & 28 & 97,87 \\
\hline P8-O2 & 64 & 0 & 0 & 29 & 100 \\
\hline Fz-Cz & 63 & 1 & 1 & 29 & 97,87 \\
\hline Cz-Pz & 64 & 0 & 2 & 28 & 97,87 \\
\hline T7-Ft9 & 62 & 2 & 1 & 28 & 96,77 \\
\hline Ft9-Ft10 & $\mathbf{6 1}$ & $\mathbf{3}$ & $\mathbf{3}$ & $\mathbf{2 7}$ & $\mathbf{9 3 , 6 2}$ \\
\hline Ft10-T8 & 63 & 1 & 2 & 27 & 96,77 \\
\hline & & & & & \\
\hline
\end{tabular}


TABLE IX

THE CLASSIFICATION RESULTS FOR PATIENT 6

\begin{tabular}{|c|c|c|c|c|c|}
\hline \multirow{2}{*}{ Channel } & \multicolumn{2}{|c|}{ normal stage } & \multicolumn{2}{c|}{ seizure stage } & \multirow{2}{*}{ Accuracy } \\
\cline { 2 - 5 } & normal & seizure & normal & seizure & \\
\hline Fp1-F7 & 64 & 0 & 0 & 28 & 100 \\
\hline F7-T7 & 64 & 0 & 0 & 28 & 100 \\
\hline T7-P7 & 64 & 0 & 0 & 28 & 100 \\
\hline P7-O1 & 64 & 0 & 0 & 28 & 100 \\
\hline Fp1-F3 & 64 & 0 & 0 & 28 & 100 \\
\hline F3-C3 & 64 & 0 & 0 & 28 & 100 \\
\hline C3-P3 & 64 & 0 & 0 & 28 & 100 \\
\hline P3-O1 & 64 & 0 & 0 & 28 & 100 \\
\hline Fp2-F4 & 63 & 1 & 1 & 27 & 97,83 \\
\hline F4-C4 & 64 & 0 & 0 & 28 & 100 \\
\hline C4-P4 & 64 & 0 & 0 & 28 & 100 \\
\hline P4-O2 & 64 & 0 & 0 & 28 & 100 \\
\hline Fp2-F8 & 62 & 2 & 0 & 28 & 97,83 \\
\hline F8-T8 & 62 & 2 & 0 & 28 & 97,83 \\
\hline T8-P8 & 63 & 1 & 1 & 27 & 97,83 \\
\hline P8-O2 & 64 & 0 & 0 & 28 & 100 \\
\hline Fz-Cz & 63 & 1 & 2 & 26 & 96,74 \\
\hline Cz-Pz & 64 & 0 & 2 & 26 & 97,83 \\
\hline T7-Ft9 & 64 & 0 & 0 & 28 & 100 \\
\hline Ft9-Ft10 & 64 & 0 & 0 & 28 & 100 \\
\hline Ft10-T8 & $\mathbf{6 1}$ & $\mathbf{3}$ & $\mathbf{1}$ & $\mathbf{2 7}$ & $\mathbf{9 5 , 6 5}$ \\
\hline & & & & & \\
\hline
\end{tabular}

The classification results show that The LDA classifier was able to perform seizure detection with error-free at least three channels for each patient. Especially for the first two patients, the classification process has been implemented without error in almost all channels. The seizure detection process affected at least level for these patients. According to the classification accuracy for patient 4 the worst performance was obtained at Fp1-F3 channel. For patient 4, the difference between accuracy of channels reached the highest level.

The first two major principal components were used to visualize the variation in performances of the classifier. In this stage, for patient 4 who would be able to affected mostly from the channel selection, $\mathrm{P} 4-\mathrm{O} 2$ channel's testing data with highest classification performance has been reflected to the plane which consist of first two major principal components. The PCA projection of this channel has been illustrated in the Figure 3.

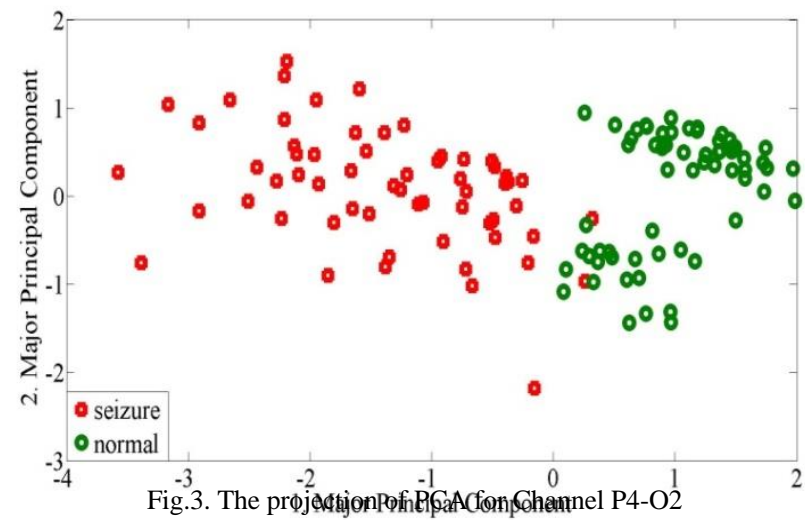

These processes has been repeated for the Fp1-F3 channel that has the lowest classification accuracy. The PCA projection for this channel has been illustrated in the Figure 4.

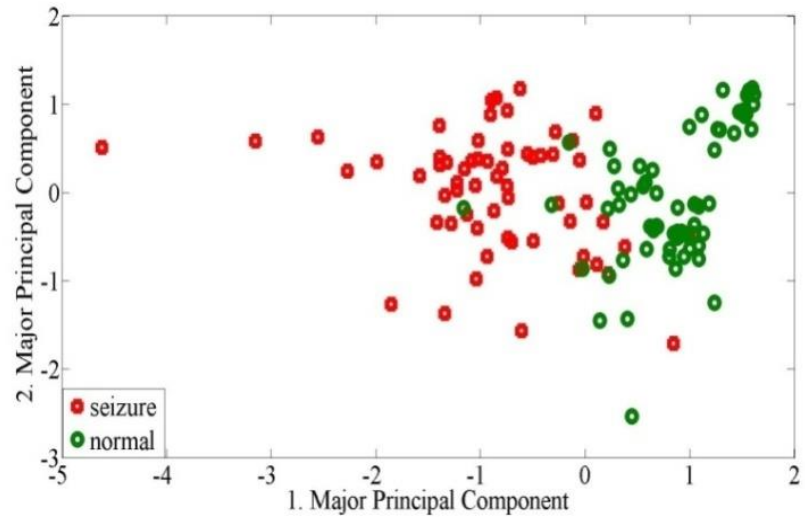

Fig.4. The projection of PCA for Channel Fp1-F3

According to Figure 3 and Figure 4, it is seen that normal and seizure stages largely separated from each other in the reflection for $\mathrm{P} 4-\mathrm{O} 2$ channel. Classification results also support the PCA projection. For this channel classification process has been performed without error. For Fp1-F3 channel, it is also seen that normal and seizure stages didn't separate clearly. These stages interfered each other. In the classification result for this channel, four normal samples detected as seizure and seven seizure samples detected as normal. Receiver Operating Characteristic curves of the classification process for these two channels are shown in the Figure 5.
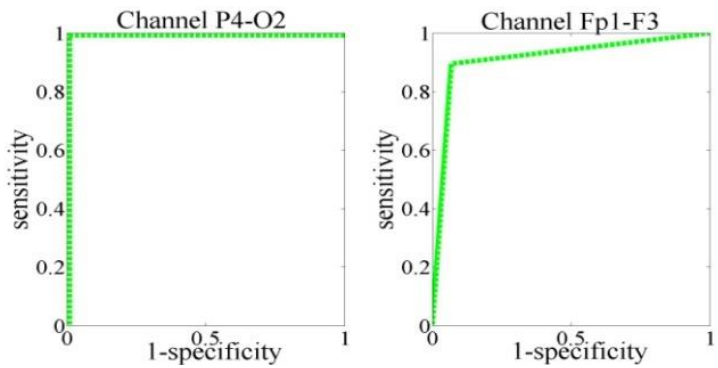

Fig.5. ROC curves for Patient 4

The area values under the ROC curve (Area Under the Curve: AUC), respectively, are calculated as 1 and 0.9133 .

\section{CONCLUSIONS}

Developments in computer, electronics, and signal processing methods began to be used widely in the medical electronics field recently. Researchers propose systems which capable of analyzing of EEG effectively. Designers have been developing portable seizure detection prototypes. A lot of processing load in the real time applications affects negatively the response speed of the seizure detector. Designers use various methods to reduce the process load to a reasonable level. In this study, it is aimed to evaluate channel selection effects on seizure detector performance by analyzing effects of EEG signals obtained from different channel on seizure detection process.

Analysis have been implemented patient-specifically for six patient. The differences between the best and worst channel 
accuracies and number of channel, is able to detect seizure with error-free, in the classification process using 21 channels for each patient are given in the Table $\mathrm{X}$.

TABLE X

THE MAXIMUM RIPPLE OF CHANNEL CLASSIFICATION ACCURACIES AND THE NUMBER OF CHANNEL WHICH IS CAPABLE OF THE ERROR-FREE CLASSIFICATION FOR EACH PATIENT

\begin{tabular}{|c|c|c|c|c|c|c|}
\hline & P1 & P2 & P3 & P4 & P5 & P6 \\
\hline $\begin{array}{c}\text { Maximum ripple of Channel } \\
\text { Accuracies }\end{array}$ & 1,08 & 1,11 & 4,00 & 8,80 & 6,38 & 4,35 \\
\hline $\begin{array}{c}\text { Number of channel capable of } \\
\text { seizure detection error-free }\end{array}$ & 19 & 20 & 9 & 3 & 7 & 14 \\
\hline
\end{tabular}

Table V shows that channel selection can affect the classification results up to \% 8.80 level. Patient 1 and Patient 2 are the most immune to channel selection. For these patients, it can be said that channel selection hasn't affected scarcely in seizure detection process. The most affected patient by channel selection was patient 4 . For this patient, classification process was performed error-free for only three channels. According to the analysis results, depending on the channel selection, a ripple at the classifier accuracy can occur about $9 \%$. These results show that Designers must choose more distinctive channels to obtain the most useful data in the channel selection processes.

\section{ACKNOWLEDGEMENT}

This research was supported by Sakarya University Scientific Research Projects Commission (Project Number: 2013-50-02010).

The study is selected from National Engineering Research Symposium 2015 (Ulusal Mühendislik Araştırmaları Sempozyumu) UMAS 2015 (Duzce University).

\section{REFERENCES}

[1] N. Sivasankari, K. Thanushkodi, "Automated Epileptic Seizure Detection in EEG Signals Using FastICA and Neural Network", Int. J. Advance, Soft Comput. Appl., Vol. 1, No. 2, pp. 1-14, 2009.

[2] A. Shoeb, J. Guttag, "Application of Machine Learning To Epileptic Seizure Detection", The 27th International Conference on Machine Learning ICML 2010, 21-24 June 2010, Haifa-Israel.

[3] H. Vavadi, A. Ayatollahi, A. Mirzaei, "A wavelet-approximate entropy method for epileptic activity detection from EEG and its sub-bands", J. Biomedical Science and Engineering, Vol. 3, No. 2010, pp. 1182-1189,2010.

[4] G. Ouyang, X. Li, Y. Li, X. Guan, "Application of wavelet-based similarity analysis to epileptic seizures prediction", Computers in Biology and Medicine, Vol. 37, No. 2007, pp. 430-437, 2007.

[5] M. Arvaneh, C. Guan, K.K. Ang, H.C. Quek, "Optimizing the channel selection and classification accuracy in EEG-Based BCI", IEEE Transactions on Biomedical Engineering, Vol. 58, No. 6, pp. 1865-1873, 2011.

[6] M. Qaraqe, M. Ismail, Q. Abbasi, E. Serpedin, "Channel Selection and Feature Enhancement for Improved Epileptic Seizure Onset Detector", 4th International Conference on Wireless Mobile Communication and Healthcare MOBIHEALTH 2014, 03-05 November 2014, Athens, Greece.

[7] J. Duun-Henriksen, T.W. Kjaer, R.E. Madsen, L.S. Remvig, C.E. Thomsen, H.B.D. Sorensen, "Channel selection for automatic seizure detection", Clinical Neurophysiology, Vol. 123 No. 2012, pp. 84-92,2012.
[8] S. Faul, W. Marnane, "Dynamic, location-based channel selection for power consumption reduction in EEG analysis", Computer Methods and Programs in Biomedicine, Vol. 108, No. 3, pp. 12061215,2012 .

[9] H. Tekgul, B.F.D. Bourgeois, K. Gauvreau, A.M. Bergin, "Electroencephalography in neonatal seizures: Comparison of reduced and a full 10-20 montage", Pediatric Neurology, Vol. 32, No. 3, pp. 155-161, 2005.

[10] PhysioNet International database, 6.12.2011 http://www.physionet.org/physiobank/database/chbmit/

[11] Meyer-Baese, Pattern Recognition for Medical Imaging, Elsevier Academic Pres, California, 2004.

[12] R.O. Duda, P.E. Hart, D.G. Stork, Pattern Classification, WileyInterscience, New York, 2001.

[13] A.K. Junoh, M.N. Mansor, "Safety System Based on Linear Discriminant Analysis", 2012 International Symposium on Instrumentation \& Measurement, Sensor Network and Automation (IMSNA), 25-28 Augost 2012, Sanya, China.

[14] N.Panahi, M.G. Shayesteh, S. Mihandoost, B.Z. Varghahan, "Recognition of Different Datasets Using PCA, LDA, and Various Classifiers", 5th International Conference on Application of Information and Communication Technologies (AICT), 12-14 October 2011, Baku, Azerbaijan.

[15] A. ÜNSAL, "Diskriminant analizi ve uygulaması üzerine bir örnek", Gazi Üniversitesi İktisadi ve İdari Bilimler Fakültesi (G.Ü.İ.I.B.F.) Dergisi, Vol.2, No. 3, pp. 19-35, 2000.

[16] A. Fielding, "Cluster and Classification Techniques for the Biosciences", Cambridge University Press, New York, 2000.

[17] M.M. Fraz, P. Remagnino, A. Hoppe, S.A. Barman, "Retinal image analysis aimed at extraction of vascular structure using linear discriminant analysis", 2013 International Conference on Computer Medical Applications (ICCMA), 20-22 January 2013, Sousse, Tunisia.

\section{BIOGRAPHIES}

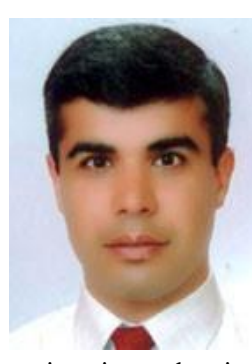

Murat YILDIZ was born in Siirt, Turkey in 1977. He received the B.Sc., M.Sc. and Ph.D. degrees in Electrical-Electronics Engineering from Faculty of Engineering, Sakarya University, Turkey in 1999, 2003 and 2009 respectively. He worked in the Dept. of Electrical-Electronics Engineering as a Research Assistant in 2000-2009, and he has been an Assist. Professor since 2009. His research areas include renewable energy (solar energy, hydrogen energy and hybrid systems), electrical and electronic systems, biomedical signal processing, biomedical engineering and optimization.

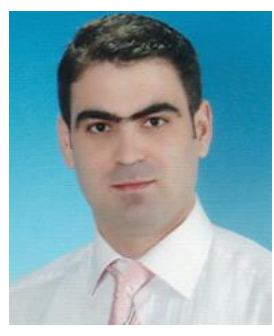

Erhan BERGiL was born in Sivas, Turkey in 1980. $\mathrm{He}$ received the B.Sc. and M.Sc. degrees in Electrical-Electronics Engineering from Faculty of Engineering, Ondokuz Mayıs University, Turkey in 2001 and 2009 respectively. He continues his Ph.D. degree in Electrical-Electronics Engineering, Sakarya University, Turkey. Between 2002-2006, he worked in the Control and Automation Program in the Vocational High School, Ondokuz Mayis University, Turkey as a lecturer. Since 2006, he has been working in the Control and Automation Technology Program, Department of Electronics and Automation, Technical Sciences Vocational School, Amasya University, Turkey, as a lecturer. His research areas include biomedical signal processing, electrical and electronic systems, control systems. 